\title{
Automated Controller Tuning for Atomic Force Microscopes Using Estimation Based Multiple Model Switched Adaptive Control
}

\author{
Umar Khan, Harold Chong and Mark French
}

\begin{abstract}
Atomic Force Microscopes (AFMs) generate topographic images with nanometer resolution and need little or no sample preparation, however their operation depends on the proper tuning of a PI controller for vertical nanopositioning. Currently these controllers need to be tuned manually by the end user which reduces their ease of use. We develop an automated online Proportional Integral (PI) controller tuning procedure for the control of vertical loop using a multiple model adaptive control (MMAC) approach. The approach is suitable for retro-fitting around an existing PI controller. Preliminary experimental results are presented.
\end{abstract}

\section{INTRODUCTION}

Ever since the development of the first Atomic Force Microscope (AFM) in 1986 by Binning et.al [1], this instrument has been widely used in a diverse range of fields ranging from material science, molecular biology to nanomanipulation and lithography. AFMs have gained popularity due to the fact that the sample can be viewed in air, vacuum or liquid and little or no sample preparation is needed. In addition the samples do not need to be conducting as was the requirement for Scanning Tunnelling Microscopes (STMs) that were used before AFMs.

Some of the most prominent examples for the usage of AFMs can be found in the fields of material science and the study of biological materials. For instance AFMs are being used for the study of material forces e.g., adhesion force [7], surface alteration as in the case of the etching processes [8], interface between materials as in the generation nanocontacts [9] and also in the study of lubrication, friction and wear [10]. AFMs have found substantial usage for the study of biological materials. This includes the study of the activity of single molecules e.g. RNA polymerase [2] [3], molecular motor motion such as Myosin V [4], cancerous cells [6] and nanomechanical analysis of cells [5]. In addition to this, the atomic force microscopy principle is not restricted to only viewing surface topography or measurement of other material properties, it can also be used for the purpose of lithography [12] and nanomanipulation [11].

The key limitation of this otherwise very versatile instrument is that its operation depends upon tuning of a controller, which is very often a simple PI (Proportional

Umar Khan is with Faculty of Physical Sciences and Engineering , University of Southampton, SO17 IBJ, United Kingdom uaaklg10@ecs.soton.ac.uk

Mark French is with Faculty of Physical Sciences and Engineering, University of Southampton, SO17 IBJ, United Kingdom mcfeecs.soton.ac.uk

Harold Chong is with Faculty of Physical Sciences and Engineering, University of Southampton, University of Southampton, SO17 IBJ, United Kingdom hmhc@ecs.soton.ac.uk
Integral) controller as in most commercially available AFMs. Currently the controllers need to be manually tuned by the end users, which can be time consuming and also requires a basic understanding of control theory fundamentals. The purpose of this contribution therefore is to report an automated PI controller tuning procedure and some preliminary experimental results obtained on an AFM.

The remaining part of this section discusses briefly the research done so far for AFM controller synthesis. Before this information can be presented a distinction must be made between the two popular modes of AFM operation namely, Contact Mode and Dynamic Mode. For the case of contact mode imaging a number of contributions have reported the use of robust adaptive, robust, nonlinear and repetitive control methods. In [13] Refai applies a robust adaptive controller for controlling an AFM in contact mode. The authors use a reduced order LTI model and presents simulation results which compare the performance of the robust adaptive controller with a well tuned PI controller. A similar approach is adopted by Sebastian[14], who first uses a system identification procedure to determine the AFM system dynamics and then applies Glover McFarlane $H_{\infty}$ procedure to design a controller. A resembling strategy has been adopted by Abramovitch [15] who assumes that the AFM dynamics are described by a second order resonant plant and then provides analytical relations for obtaining the proportional, integral and derivative gains using the parameters of the resonant plant. The resonant plant needs to be determined using a system identification procedure as described by the author.

For the case of the Dynamic Mode AFM Sambit [16] suggests a state feedback control law based on estimated state feedback and takes into account the presence of bifurcations and chaos in the Dynamic AFM mode. In [17] an external nonlinear feedback loop in addition to the standard PI control loop is suggested. Kristic [18] combines damping boundary feedback with backstepping and presents simulation results for the resulting control method. Finally, Serkan [19] suggests a Repetitive Controller which uses information from previous scan lines to improve scanning performance.

Although each of these contributions suggest suitable controller generation methods, they depend upon sophisticated controllers in addition to or in place of a simple PI controller. This research aims to develop a method that automatically tunes PI controllers used in AFMs. The primary motivation is that if such a tuning method is developed, it can be integrated with existing commercial AFMs which already have PI controllers built into them. The method chosen for 
controller tuning is based on a particular MMAC scheme: Estimation Based Multiple Model Switched Adaptive Control [21],[22], [23], see [25] for a general survey of related MMAC schemes. The concept behind this method is that the AFM input output signals are constantly observed to determine the closest linear model using a bank of deterministic Kalman filters. Once the best match plant is determined, a predetermined controller corresponding to that plant is switched into the AFM control loop. The reason for selecting this method is that it only needs the AFMs input output signals to determines the controller gains, and has the ability to incorporate a diverse range of possible AFM experimental setups. All results reported here are for the case of Dynamic Mode AFM.

Furthermore, this method is analogous to an auto pilot used in aircraft. The objective is to reduce the expertise required and generate an all purpose tool, rather than one that requires specialist training.

The remaining part of this paper is organized as follows, Section II introduces the fundamentals of Atomic Force Microscopy from a control theoretic perspective, Section III describes Estimation Based Multiple Model Switched Adaptive Control (EMMSAC), Section IV discusses the application of the EMMSAC algorithm for AFM control, Section V provides the experimental results and the last section provides conclusions and outlines possible directions for future work.

\section{ATOMIC FORCE MICROSCOPY}

As illustrated in Figure 1, an AFM setup consists of a cantilever, a laser source, a photosensitive diode, a $Z$ Piezo actuator and a Dither piezo actuator. AFM cantilevers generally have a length of up to $250 \mu \mathrm{m}$ and are generally composed of silicon oxide, silicon nitride or pure silicon [20]. The cantilever has a sharp pyramid shaped tip which is meant to probe the surface.

The front top end of the cantilever is illuminated with a laser beam generated from the laser source. This is then reflected and detected by the photo sensitive detector. This mechanism provides a means for detecting the vertical deflection of the cantilever tip.

The base of the cantilever is connected to the dither and $\mathrm{Z}$ piezos as illustrated in Figure 1. The dither piezo is excited with a sinusoidal drive voltage signal $f_{D}$. This results in a sinusoidal cantilever deflection $d_{T M}$. This deflection signal passes through a lock-in amplifier to extract the amplitude of deflection $A$.

The operation of the AFM then relies on the fact that an increase in the sample topography e.g. a bump will cause a reduction in the cantilever deflection amplitude. Likewise, a reduction in sample topography e.g., a valley will cause an increase in deflection amplitude.

The sample image is then generated by rastering the entire cantilever piezo actuator assembly over the sample surface. This is done by using separate piezos attached to the cantilever piezo assembly that enable it's lateral movement. Alternatively, the same effect can achieved by placing the sample on a piezo actuated stage and rastering it beneath the vibrating tip. The AFM used in this contribution uses the former arrangement.

As the sample topography varies, the control loop regulates the deflection amplitude $A$ at it's setpoint value $A_{S P}$. This is done by passing the error signal $e=A_{S P}-A$ to the controller and the resulting control signal to the $\mathrm{Z}$ piezo actuator through the piezo amplifier. A reduction in deflection amplitude will cause the $\mathrm{Z}$ piezo to move the cantilever base away from the sample, thus increasing the deflection amplitude to it's set point and vice versa. The controller signal $h$ then serves as an estimate of the sample topography.

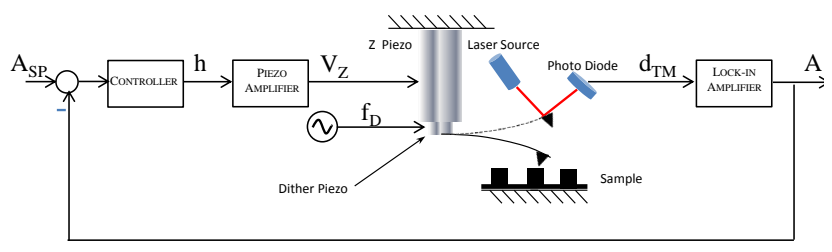

Fig. 1. Atomic Force Microscope setup.

\section{ESTIMATION BASED MULTIPLE MODEL ADAPTIVE CONTROL}

This section explains the functioning of the Estimation based Multiple Model Switched Adaptive (EMMSAC) [21],[22], [23] control algorithm. Section III-A explains the three building components of this method followed by the algorithm itself in section III-B.

\section{A. EMMSAC Components}

The basic concept behind the EMMSAC control method is that the control action is performed on the basis of a number of candidate plant representations in a set referred to as the plant model set. The complete EMMSAC algorithm can be split up into the following three parts,

Plant Model Set: The pant set $\mathscr{G}$ is a set of $N$ plants,

$$
\mathscr{G}=\left\{G_{1}, G_{2}, \ldots G_{p} \ldots, G_{N}\right\}
$$

where $N$ is the number of plants, and $1 \leq p \leq N$. The use of a plant set corresponds to the presence of structured uncertainty in the model of the true plant $G^{*}$. Each plant is an operator:

$$
G_{p}: U_{e} \rightarrow Y_{e}: u_{1} \mapsto y_{1}, 1 \leq p \leq N
$$

Here $u_{1} \in U_{e}, y_{1} \in Y_{e}$ are plant input and output signals, throughout this paper taken to be $l^{2} . U_{e}$ and $Y_{e}$ are corresponding extended signal spaces. Extended signal spaces are defined as those spaces in which signals are permitted to grow unboundedly.

Controller Set: The controller set $\mathscr{C}$ consists of $N$ controllers, each corresponding to a plant in the plant model set as specified by a design map $\mathscr{K}: \mathscr{G} \rightarrow \mathscr{C}, \mathscr{K}\left(G_{p}\right)=C_{p}$. 
Each closed loop corresponding to a matched plant controller $\left[G_{p}, \mathscr{K}\left(G_{p}\right)\right]$ is required to be gain stable.

Kalman Filter Set: The Kalman filter set contains $N$ Kalman filters, one corresponding to each of the plants in the plant set. Each filter evaluates the true plant's $\left(G^{*}\right)$ measured input and output signals namely $w_{2}=\left[u_{2}, y_{2}\right]^{T}$ respectively at each time instant $k$ to provide a residual $r_{p}(k)$. The residual has the interpretation as the size of the smallest disturbances $\left[u_{0}^{p}, y_{0}^{p}\right]^{T}$ which are compatible with the associated plant $G_{p}$ and the observations $w_{2}=\left[u_{2}, y_{2}\right]^{T}$ up to the current time $k$, see below. The exact relations for the deterministic Kalman filter and the above properties are classical, and can be found e.g. in [24].

\section{B. The EMMSAC Algorithm}

Before a controller can be switched into the control loop, it needs to be determined which plant in the plant model set is closest to the true plant $G^{*}$. This is achieved through a plant closeness function $E$. At each time step $k$, this function takes as argument the true plants measured input output signals $u_{2}, y_{2}$ and the candidate plant's $\left(G_{p}\right)$ index $p$ and returns a nonnegative scalar value $\kappa$. By convention used in this contribution the lower the value of $\kappa$ the closer is the candidate plant to the true plant.

The closeness function operates using the Disturbance Estimation Principle. This principle can be understood in the following manner. Let $w_{o}=\left[u_{o}, y_{o}\right]^{T}$ be the input and output disturbances acting on the true plant $G^{*}$ as illustrated in Figure 2. The disturbance estimation principle then estimates the closeness of $G_{p}$ to $G^{*}$ be measuring the size of the smallest disturbance signals $w_{o}=\left[u_{o}^{p}, y_{o}^{p}\right]^{T}$ which are are consistent with the plant $G_{p}$ and the same observations $\left(\left[u_{2}^{p}, y_{2}^{p}\right]^{T}=\left[u_{2}, y_{2}\right]^{T}\right)$. The consistency condition holds true if the following relations are satisfied,

$$
y_{o}^{p}-y_{2}=G_{p}\left(u_{o}^{p}-u_{2}\right)
$$

In this contribution, the size of the disturbance signals is measured in terms of the $l_{2}$ norm. The closeness function can thus be written as,

$$
E\left(w_{2}\right)(k)(p)=\inf \left\{\kappa \geq 0 \mid \kappa=\left\|v_{o}\right\|_{2}, v_{o} \in \mathscr{N}_{p}^{[0, k]}\left(w_{2}\right)\right\}
$$

Here, $\mathscr{N}_{p}^{[0, k]}\left(w_{2}\right)$ is the set of disturbance signals weakly consistent to the plant $G_{p}$ and the measured true plant input output signals $w_{2}=\left[u_{2}, y_{2}\right]^{T}$. These signals are defined as follows[24],

Definition 1: Let $k \in \mathbb{N}$. The set of weakly consistent disturbance signals $N_{p}^{[0, k]}\left(w_{2}\right)$ to a plant $P_{p}, p \in P$ and the observation $w_{2}=\left(u_{2}, y_{2}\right)^{T}$ is defined by,

$$
\mathscr{N}_{p}^{[0, k]}\left(w_{2}\right):=\left\{\begin{array}{l|l}
\left.v \in W\right|_{[0, k]} & \begin{array}{c}
\exists\left(u_{o}^{p}, y_{o}^{p}\right)^{T} \in W_{e} s . t \\
\mathscr{T}_{k} P_{p}\left(u_{1}^{p}\right)=\mathscr{T}_{k}\left(y_{1}^{p}\right), \\
\left.v=\mathscr{T}_{k} u_{o}^{p}, \mathscr{T}_{k} y_{o}^{p}\right)
\end{array}
\end{array}\right\}
$$

Here $W_{e}=U_{e} \times Y_{e}$ and $\mathscr{T}_{k}$ is the truncation operator:

$$
\mathscr{T}_{k} w=\left\{\begin{array}{ll}
w(s) & 0 \leq s \leq k \\
0 & s>k
\end{array} .\right.
$$

As above, the size of the smallest $l_{2}$ norm disturbance signal $v_{o}$ for $G_{p}$ can be determined efficiently by evaluating the residual $r_{p}(k)$ for the corresponding Kalman filter using the $G^{*}$ 's measured input output signals thus giving,

$$
E\left(w_{2}\right)(k)(p)=r_{p}(k)
$$

This residual is evaluated for all plants in the set using the bank of Kalman filters. As mentioned previously each filter evaluates the true plant's $\left(G^{*}\right)$ measured input and output signals namely $w_{2}=\left[u_{2}, y_{2}\right]^{T}$ respectively at each time instant $k$ to provide a residual $r_{p}(k)$. Further details regarding this proceedure can be found in [24]. These residuals are used to generate a switching signal $q$ that always points to the plant with the least residual,

$$
q(k):=\arg \min _{1 \leq p \leq N} r_{p}[k], \forall k \in \mathbb{N}
$$

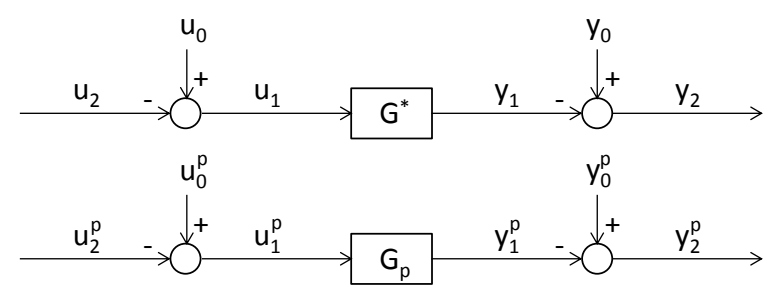

Fig. 2. Disturbances and observations.

The complete EMMSAC control loop is illustrated in Figure 3. Here the aregument $w_{2}$ and $k$ has been dropped from $E\left(w_{2}\right)(k)(p)$ for clarity.

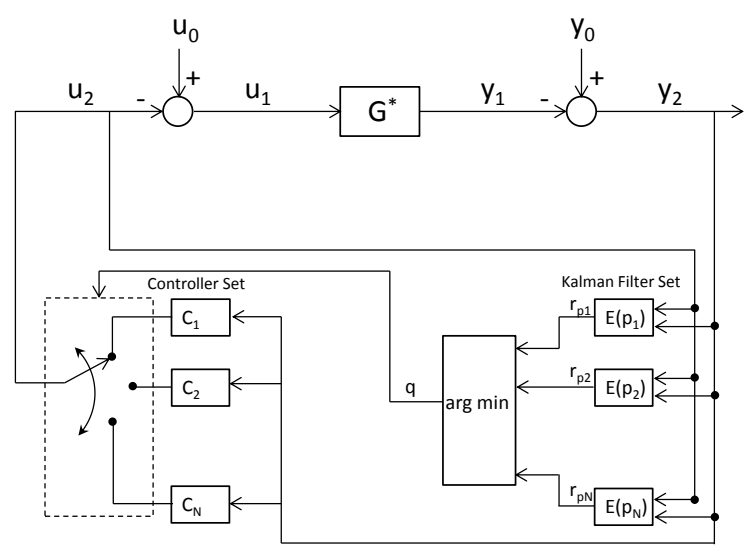

Fig. 3. The complete EMMSAC algorithm.

\section{APPLICATION OF EMMSAC FOR AFM CONTROL}

All experiments in this contribution have been done on the Nanonics Imaging CryoView 2000 AFM. The cantilever obtained from Nanosensors is made of n-silicon with a length of $240 \mu \mathrm{m}$. The resonance frequency and quality factor as measured through a frequency sweep experiment are $65.5 \mathrm{kHz}$ and 180.4 respectively. Two samples are scanned namely, the TGZ 02 calibration grid from Micro Masch and 
a DVD (Digital Video Disc) sample. The calibration grid contains rectangular $\mathrm{SiO}_{2}$ steps on $\mathrm{Si}$ wafer with a step height of $120 \pm 0.5 \mathrm{~nm}$ and a pitch of $3.0 \mu \mathrm{m}$. In each case an area of $3 \times 3 \mu \mathrm{m}$ is scanned.

The implementation of the EMMSAC algorithm is illustrated in Figure 4.

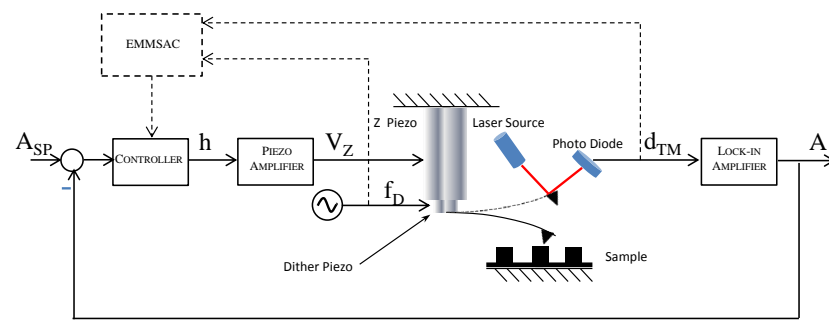

Fig. 4. Implementation of the EMMSAC algorithm with the AFM in the loop.

In this case $u_{2}=\left[h, f_{D}\right]^{T}, y_{2}=d_{T M}$. The output disturbance $y_{0}$ principally originates from the sample topography. The AFM plant is a Multiple Input Single Output (MISO) plant. Ideally the plant set for EMMSAC implementation should therefore have the same structure. However given the nonlinear relationship between the $h$ and $d_{T M}$, this contribution attempts to implement the EMMSAC algorithm by considering only the $f_{D}$ input. The relation between $f_{D}$ i.e the dither piezo input and $d_{T M}$ the cantilever deflection is linear and can be approximated by a second order transfer function, as has been reported in [27].

At every time step while the vibrating cantilever is being rastered over the sample, $f_{D}$ and $d_{T M}$ are recorded at a rate of $10^{6}$ samples/second using a National Instruments 6356 USB DAQ. Ideally the EMMSAC algorithm should be run after the completion of each scan line, the least residual plant should be determined and the corresponding controller should be switched into the loop for the next scan line. However due the computationally intense nature of the algorithm this process is repeated only three times during the entire sample scan. Each scan consists of 512 lines, 256 trace lines and 256 retrace lines. The remaining part of this section explains the generation of the plant set and the corresponding controller set. The PI controller is implemented on the TMS320C6713 DSK from Texas Instruments using 16 bit $\mathrm{A} / \mathrm{D}$ and $\mathrm{D} / \mathrm{A}$ converters.

Plant Set: The plant set is generated by test a set of trial PI controllers in the set $C_{T}$ given below,

$$
\left(K_{p}, K_{i}\right) \in C_{T}=[0,0.1,0.2, \ldots, 1.0] \times[0,10,20, \ldots, 100]
$$

Here $K_{p}$ and $K_{i}$ represent the proportional and integral gains of the PI controller used in the AFM control loop. The range and separation of the gains is chosen empirically. This combination of $\mathrm{P}$ and I gains results in 121 controllers in the trial controller set $C_{T}$.
The plant set is determined through a series of six system identification experiments, three for each sample. The reason for doing a relatively large number of experiments is to include the variations in the AFM plant (piezo actuator, cantilever dynamics and changes in ambient operating conditions). In each experiment all the controllers given in the trial set $C_{T}$ are tested twice, once for a trace line and once for a retrace one. The terms trace and retrace reffer to consecutive scan lines in a raster scan pattern. Each scan line takes one second to execute. During this scan four signals are recorded namely $A, h, f_{D}, d_{T M}$. The testing of each controller generates two data sets $D_{j T}^{i}$ and $D_{j R}^{i}$ given below,

$$
\begin{aligned}
& D_{j T}^{i}=\left[\begin{array}{lll}
A h f_{D} & d_{T M}
\end{array}\right] \in \mathbb{R}^{10^{6} \times 4} \mid 1 \leq j \leq 121,1 \leq i \leq 6 \\
& D_{j R}^{i}=\left[\begin{array}{lll}
A h f_{D} & d_{T M}
\end{array}\right] \in \mathbb{R}^{10^{6} \times 4} \mid 1 \leq j \leq 121,1 \leq i \leq 6
\end{aligned}
$$

Here $j$ refers to the controller being tested, $i$ equals the experiment number and the subscripts $\mathrm{T}$ and $\mathrm{R}$ correspond to trace and retrace lines respectively. Next, the dither piezo input $f_{D}$ and cantilever deflection $d_{T M}$ in each data set $D_{j}^{i}$ are used to determine the closest second order LTI plant $G_{j}^{i}$ given below,

$$
G_{j}^{i}=\frac{k_{o} \omega_{n}^{2}}{s^{2}+\frac{\omega_{n}}{Q} s+\omega_{n}^{2}}
$$

Here $k_{o}$ is the system gain, $w_{n}$ is the resonance frequency in $\operatorname{rads}^{-1}$ and $\mathrm{Q}$ is the quality factor. The choice of this structure is motivated from the fact that the transfer function from the piezo input voltage to the cantilever deflection can be well approximated by a second order LTI system. Given a data set $D_{j}^{i}$ the corresponding observations $w_{2}=\left[f_{D} d_{T M}\right]$. The closest plant $G_{j}^{i}$ corresponding to these observations is determined by using the closeness function $E\left(w_{2}\right)(k)(p)$. However since no apriori plant grid exists the index variable $p$ is meaningless. The closeness function is therefore reformulated, and the index variable $p$ is replaced by $P_{j}^{i}$,s parameters $Q, \omega_{n}, k_{o}$. The closest plant can then be found by solving the following optimization problem,

$$
G_{j}^{i}=\arg \min _{\left[Q \omega_{n} k_{o}\right]} \sum_{k=1}^{k=10^{6}} E\left(w_{2}\right)(k)\left(\left[Q \omega_{n} k_{o}\right]\right)
$$

subject to the constraints $0<Q \leq 500,2 \pi(1000)<\omega_{n} \leq$ $2 \pi\left(10^{5}\right)$, and $0<k_{o} \leq 100$. The optimization is performed using the Simplex search algorithm. The final plant set $\mathrm{P}$ can therefore be represented in the following manner,

$$
G=\left[\begin{array}{cccccc}
G_{1 T}^{1} & \ldots & G_{121 T}^{1} & G_{1 R}^{1} & \ldots & G_{121 R}^{1} \\
G_{1 T}^{2} & \ldots & G_{121 T}^{2} & G_{1 R}^{2} & \ldots & G_{121 R}^{2} \\
\vdots & \vdots & \vdots & \vdots & \vdots & \vdots \\
G_{1 T}^{6} & \ldots & G_{121 T}^{6} & G_{1 R}^{6} & \ldots & G_{121 R}^{6}
\end{array}\right]
$$

Here each column corresponds to the testing of a single controller and each row corresponds to one system identification experiment. The following figure illustrates the 


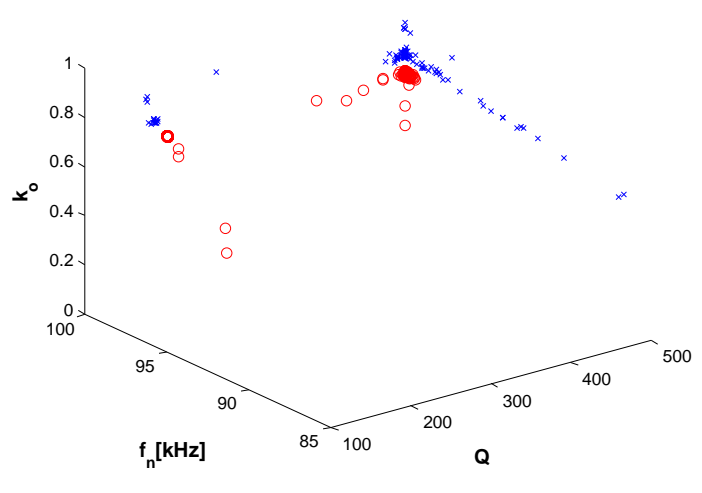

Fig. 5. Plant set obtained for experiment one and two. (Blue ' $x$ ':TGZ Red 'o':DVD)

plant set obtained from a selected experiments, in this case experiments 1 and 2 .

These experiments (1 and 2$)$ were performed for the TGZ 02 calibration and the DVD samples respectively. As can be seen, the plants corresponding to the two sample cluster at slightly different locations, however there is considerable overlap as well.

\section{Controller Set}

The system identification data is used once again to select the best controller for each experiment. This is done by evaluating the error signal $e=A_{s p}-A$ for each controller test in an experiment. The performance metric $\chi$ for each controller is then given below,

$$
\chi=\sum_{k=1}^{10^{6}} e_{T}^{2}(k)+\sum_{k=1}^{10^{6}} e_{R}^{2}(k)
$$

Here $e_{T}$ is the error for the trace line and $e_{R}$ is the error for the retrace line. For each experiment the controller with the lowest $\chi$ is selected. However three constraints are applied. Firstly, controllers that result in a large difference between trace and retrace height signals are discarded. Secondly, controllers that cause significant oscillations in the height signal are also discarded. Finally, controllers that result in height signals that are nearly flat i.e., indicating no interaction with the sample are also discarded. These constraints are imposed by two metrics given below,

$$
T R=\sum_{k=1}^{10^{6}}\left(h_{T}(k)-h_{R}(k)\right)^{2}
$$

where $h_{T}$ is the height signal for a trace and $h_{R}$ is the height signal for a retrace line. A higher value of TR indicates greater mismatch between trace and retrace lines. In addition a very low value implies that the height signals are nearly flat and there is no significant interaction between the cantilever and the sample.

The second metric PSD is the power spectral density of the height signals for frequencies greater than $10 \mathrm{~Hz}$.
This frequency was chosen empirically based on the apriori knowledge of the sample topographies used in this contribution and the scan speed. If a controller results in a height signal with substantial energy in frequencies above $10 \mathrm{~Hz}$, this implies that the gains are too high and are producing erroneous height signals. Likewise, a very low PSD value again indicates flat height signals and therefore no significant interaction between cantilever and sample. The measure is given below,

$$
P S D=\sum_{f=10}^{500000}\left|H_{T}(f)\right|^{2}+\sum_{f=10}^{500000}\left|H_{R}(f)\right|^{2}
$$

Here $H_{T}$ and $H_{R}$ are the Fast Fourier Transforms of the trace and retrace height signals respectively. The upper frequency limit in the PSD calculation i.e. $500,000 \mathrm{~Hz}$ is chosen to be half of the signal sampling frequency of the DAQ card.

Finally a metric matrix $\Phi$ is constructed for each experiment as follows,

$$
\Phi^{i}=\left[\begin{array}{cccc}
\chi_{1} & \chi_{2} & \ldots & \chi_{121} \\
T R_{1} & T R_{2} & \ldots & T R_{121} \\
P S D_{1} & P S D_{2} & \ldots & P S D_{121}
\end{array}\right]
$$

Here the superscript $i$ indicates the experiment number. Now the best controller for the experiment is chosen using the following relation,

$$
C^{i}=\arg \min _{j} \chi_{j} \mid \begin{gathered}
T R_{L}<T R_{j}<T R_{U} \\
P S D_{L}<P S D_{j}<P S D_{U}
\end{gathered} \forall 1 \leq j \leq 121
$$

here $T R_{L}, T R_{U}$ are the $5^{\text {th }}$ and $80^{\text {th }}$ percentile for all TR values in the $i^{\text {th }}$ experiment. Likewise $P S D_{L}, P S D_{U}$ are the $5^{\text {th }}$ and $30^{\text {th }}$ percentiles for all PSD values in the $i^{\text {th }}$ experiment. These limits are chosen empirically and are meant to discard controllers with unacceptable performance.

The final controller set then consists of six controllers one corresponding to each experiment and is illustrated in Table I.

TABLE I

CONTROLler SET.

\begin{tabular}{|c||c||c|}
\hline Exp. No. & $K_{p}$ & $K_{i}$ \\
\hline 1 & 0.2 & 9 \\
\hline 2 & 0.9 & 7 \\
\hline 3 & 0.1 & 10 \\
\hline 4 & 0.5 & 9 \\
\hline 5 & 0.7 & 5 \\
\hline 6 & 0.2 & 6 \\
\hline
\end{tabular}

\section{EXPERIMENTAL RESULTS}

This section presents the imaging results obtained after the execution of the EMMSAC algorithm for the two samples, mentioned in the previous section. Each scan covers and area of $3 \times 3 \mu \mathrm{m}$ and the scans are carried out at a rate of $0.5 \mathrm{~Hz}$ i.e. each scan line takes one second for completion. Due 
the computationally intensive nature of the EMMSAC, the algorithm is run at three uniformly spaced intervals during the scan starting immediately after the first line. During each EMMSAC run, the dither piezo input $f_{D}$ and the cantilever deflection signal $d_{T M}$ for a single trace line are saved. Next the residuals for all the plants given in the plant set $G$ (Equation 14) are evaluated for the recorded signals $f_{D}$ and $d_{T M}$. The switching signal then equals the row which has the least residual plant. Finally the controller corresponding to the switching signal is put into the AFM control loop and is allowed to stay in the loop until the start of the next consecutive EMMSAC run. During each EMMSAC run a total of $242 \times 6$ plant residuals are evaluated. This requires 4 minutes and 50 second on an Intel $2.67 \mathrm{GHz}$ desktop. During this interval while the residuals are being computed the AFM scan is halted. The generation of the complete image requires approximately 27 minutes.

Figures 6 and 8 illustrate the images obtained and the switching signal after each EMMSAC run. The TGZ 02 calibration sample consists of $\mathrm{SiO}_{2}$ steps on $\mathrm{Si}$ wafer with a height of $120 \mathrm{~nm}$ and pitch of $3 \mu \mathrm{m}$. In Figure 6 a single step can be viewed. Ideally a greater scan area is preferable to enable viewing of a greater number of steps, however the current AFM instrument has a maximum permissible scan area of approximately $3 \times 3 \mu \mathrm{m}$. Figure 8 reveals the tracks on a DVD sample with a spacing of approximately $0.74 \mu \mathrm{m}$ as mentioned in [26]. The height of the DVD tracks is 100 $\mathrm{nm}$ as measured using a second AFM instrument.

Figures 7 and 9 illustrate the sample heights for a single trace and retrace line. The height signals are chosen for illustrating the results since the sample feature heights are a priori known and hence serve as a bench mark. As can be seen, that although there is still a small amount on noise present in the system, the topography for the TGZ 02 sample has an error of approximately $20 \mathrm{~nm}$,the trace and retrace signals are fairly similar and the sample topography is clearly revealed. As illustrated the sixth controller is chosen twice for the TGZ calibration sample followed by the second controller. In the case of the DVD sample only the second controller is chosen. The fact that the sixth controller is sufficient for two thirds of the TGZ sample, and the second controller for the entire DVD sample is because of the relatively constant sample properties and imaging conditions.
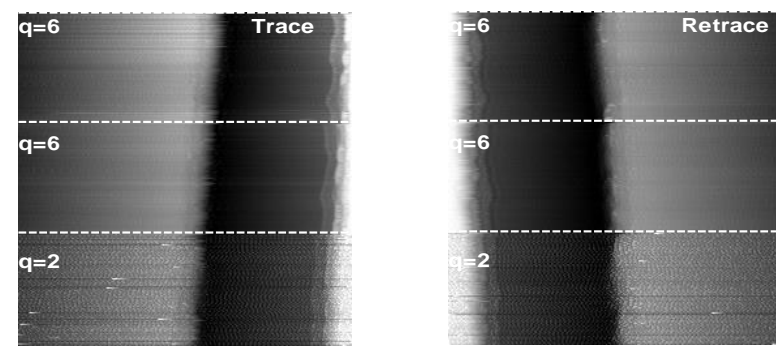

Fig. 6. TGZ calibration sample image.

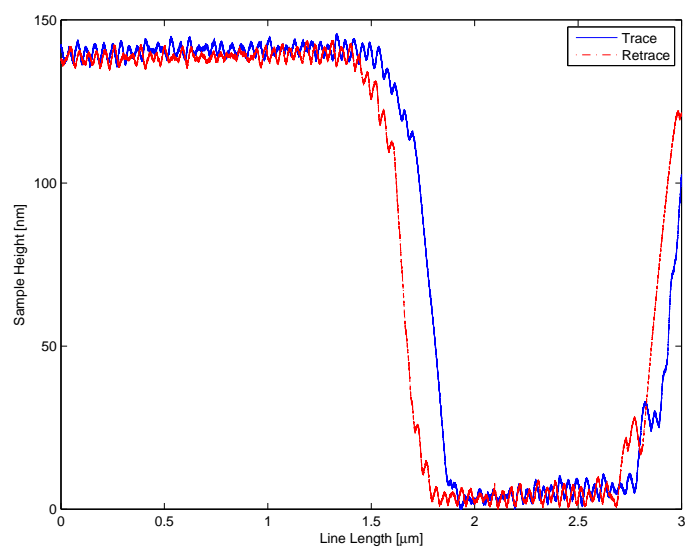

Fig. 7. TGZ calibration sample line image.
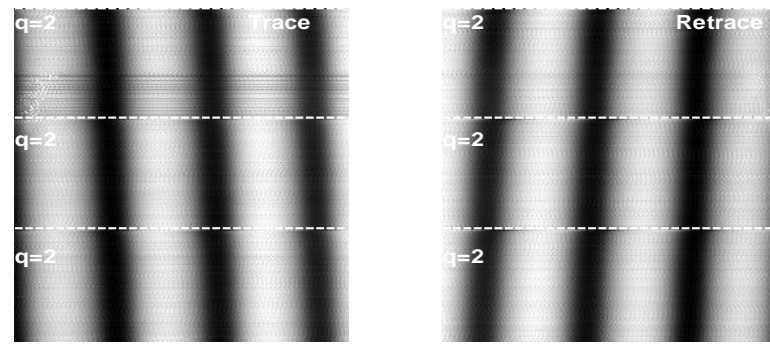

Fig. 8. DVD sample image.

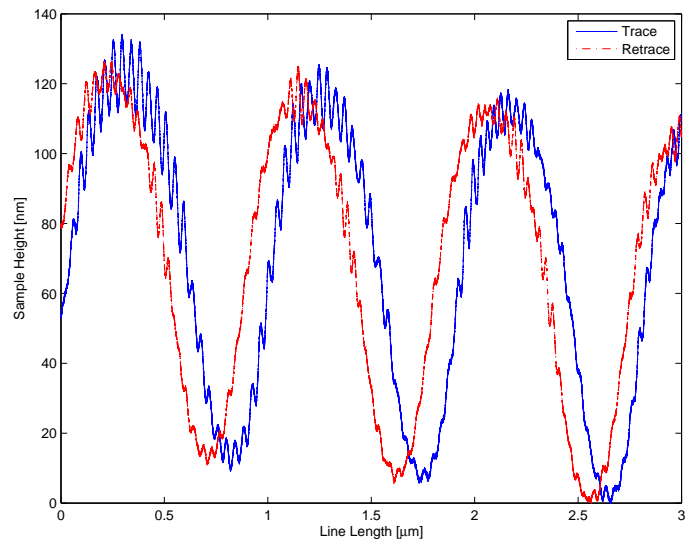

Fig. 9. DVD sample line image.

\section{CONCLUSIONS}

The objective of this contribution is to propose an automated tuning method for the PI controller gains in an AFM. As has been demonstrated through the experimental results reported here, that the EMMSAC algorithm is able to tune the controller gains without human intervention and produce suitable images. The algorithm needs to be optimized further so that it has a reduced execution time and can work with greater number of plant models and controllers thus incorporating greater robustness and improving performance; in addition enabling the running of the estimators in real 
time, leading to a continuous operation. The imaging time can be reduced by running the rastering piezos for the sample stage using a closed loop control mechanism. In addition the proposed method needs to be tested in drastically different environments for instance vacuum and liquid environments. The same method can be extended to other forms of scanning probe microscopy which have similar controller tuning requirements. This preliminary research has the potential to eventually eliminate the need for manual parameter tuning of these instruments, thus making them accessible to a greater number of users.

\section{REFERENCES}

[1] G. Binning, C.F. Quate and Ch. Gerber "Atomic Force Microscope", in Physical Review Letters, 1986, pp. 930.

[2] M. Guthold, M. Bezanilla, D.A. Erie, B. Jenkins, H.G. Hansama and C. Bustamante, "Following the assembly of RNA polymeraseDNA complexes in aqueous solutions with the scanning force microscope,1994,pp.12927 - 12931.

[3] S. Kasas, H. H. Thomson, B.L. Smith, H.G. Hansama, X. Zhu, M. Guthold, C. Bustamante, E. T. Kool, M. Kashlev and P. K. Hansama,'Escherichi Coli RNA polymerase activity observed using atomic force microscopy, 1997,pp.461-468

[4] N. Kodera, D. Yamamoto, R. Ishikawa and T. Ando, "Video imaging of walking myosin V by high-speed atomic force microscopy", in Nature, 2010, pp.72-76

[5] R. Yang, N. Xi, K. W. Lai, B. Zhong, C. K. Fung, C. Qu and D. H. Wang, "Nanomechanical analysis of insulinoma cells after glucose and capsaicin stimulation using atomic force microscopy", in Acta Pharmacologica Sinica, 2011, pp. 853-860

[6] S. Iyer, R. M. Gaikwad, V. Subba-Rao1, C. D. Woodworth and I. Sokolov, "Atomic force microscopy detects differences in the surface brush of normal and cancerous cells", in Nature Nanotechnology, 2009, pp. 389-393

[7] O. Dos, S. Ferreiraa, E. Gelinckb, D. de Graafb and H. Fischer, "Adhesion experiments using an AFMParameters of influence", in Applied Surface Science, 2010, pp. 48-55.

[8] Y. Morita, S. Migitaa, W. Mizubayashia and H. Otaa, "AFM measurement of atomic-scale Si surface etching by active oxidation", in Surface Science, 2010, pp. 1432-1437

[9] M. Grodzicki, S. Smolareka, P. Mazura, S. Zubera and AntoniCiszewski, "Characterization of $\mathrm{Cr} / 6 \mathrm{H}-\mathrm{SiC}\left(\begin{array}{llll}0 & 0 & 0 & 1\end{array}\right)$ nano-contacts by current-sensing AFM", in Applied Surface Science , 2009, pp. 10141018

[10] D. Martnez-Martnez, L. Kolodziejczyk, J.C. Snchez-Lpeza and A. Fernndez, "Tribological carbon-based coatings: An AFM and LFM study", in Surface Science, 2009, pp. 973-979

[11] Q. Yang and S. Jagannathan, "Atomic force microscope based nanomanipulation with drift compensation", in International Journal of Nanotechnology, 2006, pp. 527-544

[12] W. J. Dauksher, K. J. Nordquist, N. V. Le, K. A. Gehoski, D. P. Mancini, D. J. Resnick, L. Casoose, R. Bozak, R. White, J. Csuy and D. Lee, "Repair of step and flash imprint templates", in Journal of Vacuum Science and Technology B: Microelectronics and Nanometer Structures , 2004, pp. 3306-3311

[13] O. M. El Refai and K. Y. Toumi, "On Automating Atomic Force Microscopes: An Adaptive Control Approach", in 43rd IEEE Conference on Decision and Control, 2004.

[14] A. Sebastian, "Nanotechnology: A Systems and Control Approach", Ph.D. Dissertation, Depatrment of Electrical Engineering, Iowa State University, 2004.

[15] D. Y. Abramovitch, S. Hoen, R. Workman,'Semi-Automatic Tuning of PID Gains for Automatic Force Microscopes", in 2008 American Control Conference, 2008.

[16] S. Misra, H. Dankowicz and M.R. Paul, "Event-driven Feedback Tracking and Control of Tapping-mode Atomic Force Microscopy", in Proceedings of the Royal Society, 2008.

[17] M. Basso, P. Paoletti, B. Tiribilli and M. Vassalli, "AFM Imaging via Nonlinear Control of Self Driven Cantilever Oscillations", in IEEE Transactions On Nanotechnology, 2011.
[18] M.Krstic, A. Balogh and A. Smyshlyaev, "Backstepping Boundary Controllers for Tip-Force Induced Flexible Beam Instabilities Arising in AFM", Proceedings of the 45th IEEE Conference on Decision and Control, 2006.

[19] S. Necipoglu, S. A. Cebeci, Y. E. Has, L. Guvenc and C. Basdogan, "A Robust Repititive Controller for Fast AFM Imaging", in IEEE Transactions On Nanotechnology

[20] Olympus,"Micro Cantilever -Product Line",[Online]. Available:http://probe.olympusglobal.com/en/product/image/productline.pdf

[21] D. Buchstaller, M. French, "Scaling of Gain Bounds for Switched Adaptive Control with Large Uncertainities", IEEE Conference on Decision and Control,2007.

[22] D. Buchstaller, M. French, "Gain Bounds for Multiple Model Switched Adaptive Control of General MIMO LTI Systems", IEEE Conference on Decision and Control,2008.

[23] D. Buchstaller, M. French, "Robust Stability and Performance Analysis for Multiple Model Adaptive Controllers", IEEE Conference on Decision and Control,2009.

[24] D. Buchstaller, "Robust Stability and Performance Multipe Model Switched Adaptive Control", Ph.D. Dissertation,Department of Electrical and Computer Engineering, University of Southampton, 2010.

[25] S. Fekri, M. Athans and A. Pascoal, "Issues, progress and new results in robust adaptive controll", International Journal of Adaptive Control and Signal Processing, vol.20, pp. 519579, 2006.

[26] K. Tatebe, "Optical Data Storage", [Online]. Available:budker.berkeley.edu/Physics208/

[27] R. Garcia, A. S. Pauloi, "Dynamics of a vibrating tip near or in intermittent contact with a surface", in Physical Review B,vol. 61, no. $20,2000$. 